\title{
A Study on Piperine, Active Compound of Black Pepper
}

\author{
Faik Gökalp \\ Kırıkkale University, Faculty of Education, Department of Primary Education, Science Education \\ Division, Yahşihan/Kırıkkale, Turkey
}

Received Date: 2016-07-29 Accepted Date: 2016-10-13

\begin{abstract}
Piperine, the most important compound of black pepper, is used for treating several diseases such as breast, colon, rectal, and stomach cancer. In this study, the thermodynamical properties of this molecule in blood were obtained by using DFT and HF at the level of B3LYP/6-31+g(d,p). The results of calculations are supported with the experimental literatüre knowledges. DFT and HF basis set calculations support to the each other as comparing theoretical calculations of the interactions taking place within the molecular species
\end{abstract}

Keywords: Piperine, DFT and HF

\author{
Abbreviations \\ DFT density functional theory \\ HF hartree-fock \\ HOMO highest occupied molecular orbital \\ LUMO lowest unoccupied molecular orbital \\ FDA the food and drug administration \\ CYP3A4 Cytochrome P450 3A4
}

\section{Introduction}

Black pepper is mainly used for meals among the spices. It is worthy for its several bitter qualities based on the alkaloid structure of piperine. It is used not only in cooking but also for a lot of aims for example, medicinal, preservative, and perfumery field [1].

Piperine are alkaloid-amide components obtained from Piper species. Several of them have been reported to be cytotoxic activity towards tumor cell lines [2]. Piperine has been reported to display central nervous system depressant, antipyretic, analgesic, and anti-inflammatory activities. Piperine also protects against chemical carcinogens [3]. Moreover, piperine is a potent inhibitor of the mixed function oxygenase system and of P450 isoenzymes [4].

Modern man is confronted with an increasing incidence of cancer and cancer deaths annually. Statistics indicate that men are largely plagued by lung, colon, rectal, and prostate cancer, while women increasingly suffer from breast, colon, rectal, and stomach cancer [5]. The literature indicates that many natural products are available as chemoprotective agents against commonly occurring cancer types [6]. 'Docetaxel is the primary treatment confirmed by the FDA for prostate cancer. It is metabolized in the liver by hepatic CYP3A4 activity. Piperine, a major plant alkaloid, has been shown to inhibit the CYP3A [2], enzymatic activity in a cell-free system. In a study, it was observed piperine and docetaxel could increase docetaxel's pharmacokinetic activity in vitro and in vivo.Docetaxel is one of the most commonly used cytotoxic chemotherapeutic agents. Dietary constituents are important agents modifying drug metabolism and transport. Dietary consumption of piperine increases the therapeutic efficacy of docetaxel in a xenograft model without inducing more adverse effects on the treated mice' [7].

Piperine is an active component of black pepper. It has antimutagenic potential against carcinogens [8] In vivo, the effect of piperine on serum and tissue glycoprotein levels benzopyrene initiated lung carcinogenesis in Swis albino mice. Piperine, acts as an antioxidant and anticancer agent, was found to be chemoprotective effective, especially in lung cancer [9]. Piperine has been effected inhibition of pancreate tumor and breast cancer stem cells. It reduces cancer incidence in lung cancer [10]. It exhibits analgesic and anticonvulsant effects and important for medicinal uses of black pepper in pain and epilepsy [11]. It has treatment effects by activating Caspe-3 prostate cancer cells [12]. 
As traditional medicines become increasingly popular globally, the significant potential for interaction between traditional medicines and allopathic medicines tends to hog the limelight. Numerous studies have shown that naringin interferes with the activities of transporters and enzymatic proteins in the intestines and, hence, with the absorption and breakdown of certain drugs, resulting in altered blood levels of these drugs [13].

Güllüoğlu and his friends emphasized in their studies that the density functional methods are reliable and provide valuable information for understanding the vibrational spectra of piperidine by using a B3LYP/6-31 G (d) functional basis set [14]. Mahalakshmi and Balachandran indicated that HOMO and LUMO energies, the dipole moment of 4-Aminomethylpiperidine for the conjugative interactions and other type of interactions taking place within the molecular species had a better result compared with the experimental FT-IR and FT-Raman spectra by using B3LYP levels of theory $6-311+\mathrm{G}(\mathrm{d}, \mathrm{p})$ basis set than HF [15].

\section{Materials and methods}

The electronic structures of piperine is studied by first principles methods that contains electronic correlation and spin orbital corrections. DFT and $\mathrm{HF}$, included in. RB3LYP methods were used for geometry optimization. The correction was carried out by means of the $6-31+g(d, p)$ functional. The thermodynamical values in blood were calculated by using DFT and HF methods.

The thermodynamical values in blood were calculated by using DFT and HF method. The correction was carried out by means of the $6-31+\mathrm{g}$ (d, p) functional. The electric constant is 58 at 37 degrees Celsius for human body [16]. These methods and fully optimized geometric structure of the compounds using this method were determined and evaluated [17]. All calculations were performed using the Gaussian 09 program The structure of compound were optimized at the B3LYP/6-31G (d, p) level [18-19].

\section{Results and discussions}

The values of $\Delta \mathrm{G}$ (Gibbs free energy), HOMO, LUMO, $\Delta$ (HOMO-LUMO) and Dipol Moment releated Piperine by using DFT and HF are given in Table 1.

Table 1 The values of $\Delta \mathrm{G}$ (Gibbs free energy), HOMO, LUMO, $\Delta$ (HOMO-LUMO) and Dipol Moment releated Piperine by using DFT and HF

\begin{tabular}{llllll}
\hline $\begin{array}{l}\text { Active compound } \\
\text { of black pepper }\end{array}$ & $\begin{array}{l}\Delta \mathbf{G} \\
\text { (Hartree) }\end{array}$ & HOMO & LUMO & $\begin{array}{l}\Delta \text { (HOMO- } \\
\text { LUMO) }\end{array}$ & $\begin{array}{l}\text { Dipole } \\
\text { Moment } \\
\text { (Debye) }\end{array}$ \\
\hline $\begin{array}{l}\text { Piperine DFT } \\
\text { (In Blood) }\end{array}$ & -939.435916 & -0.20898 & -0.07290 & -0.13608 & 6.3612 \\
\hline $\begin{array}{l}\text { Piperine HF } \\
\text { (In Blood) }\end{array}$ & -933.591344 & -0.28643 & 0.06326 & -0.22317 & 5.9750 \\
\hline
\end{tabular}

The structure of Piperine is given in Figure 1.

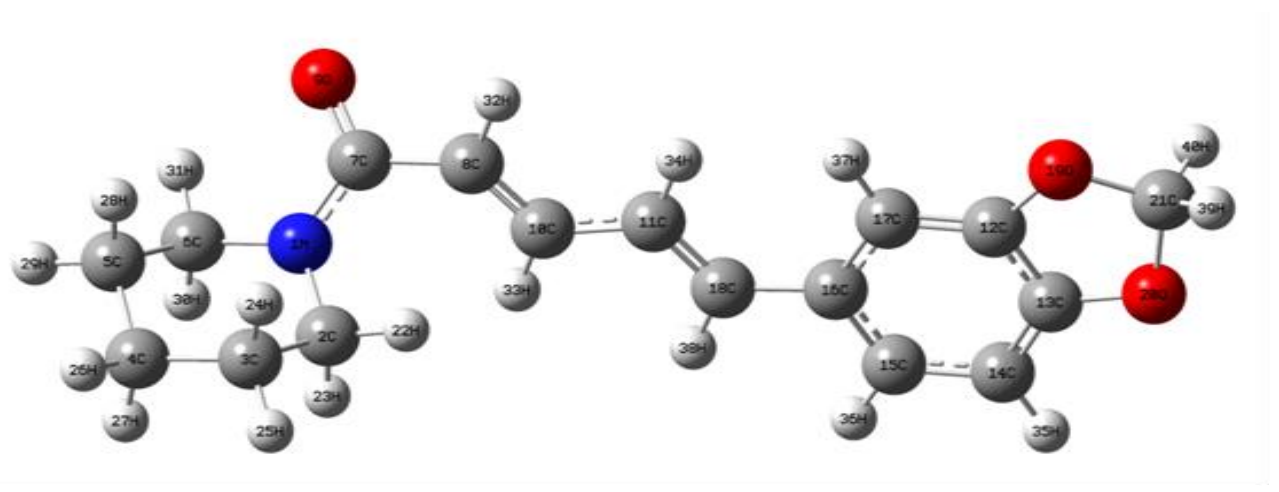

Figure 1. The structure of Piperine 
As seen in the Table 1: Free energy of Piperine, respectively, by DFT and HF, are -939.435916, 933.591344 Hartree. They are rather higher and the difference between HOMO-LUMO is -0.13608 by DFT. Dipole moments of this molecule are respectively $6.3612,5.9750$ dpi with DFT and HF.

According to the molecular orbital theory; when it occurs atoms within molecules necessary bond creates atomic orbitals mixed molecule of molecular orbitals leading to the formation they approach each other. These orbitals can be considered as one where there is greater probability of electrons in molecules [20]. The gap between HOMO and LUMO indicates the stability of the compound [21].

Ionization potential (I) is the energy to move away one e- from the molecule in the gas phase.

$\mathrm{I}=-\mathrm{EHOMO}$

The ionization potential of Piperine, in blood phase by using $\mathrm{HF}$ is $0.28643 \mathrm{eV}$. It is higher than from the other in Table 1.

Electron affinity (A) is the energy of a molecule added one e-

$\mathrm{A}=-\mathrm{ELUMO}$

The electron affinity of Piperine, in the blood by using DFT is $-0.07290 \mathrm{EV}$. It is higher than from the other in Table 1.

The datas from DFT and HF, we concluded that piperine is less stable. So, it can react easily with radicalic forms and prevent their harmful effects to the cell structures. Calculation of the polarity of the measurement of the dipole moment, considering the dipole moment vector of each bond is present in the form of the resultant moment vector [22]. He et al. emphasized that the plant's active compound were isolated with ethanol efficiently [23]. Piperine can dissolves in blood and reaches every tissue of the body because of having a high dipole moment. Wang et al. emphasized that the lone pairs of the oxygen atom in $\mathrm{Y}$-shaped oxoanions of the molecule directly interact with the $-\mathrm{NH}$ groups of the other compounds [24].

The methods for these calculations are supported in the literature $[23,24]$. As a result of this study, we can assume that piperine may be interact with the blood compounds in the similar way.

\section{Conclusions}

Experimental studies that mentioned above also indicated that positive results of these theoretical remarkably similarity. The results of these calculations indicated that piperine can dissolves in blood and reaches the every tissue of the body because of having a high dipole moment. It is less stable. So, it can react easily with radicalic forms and decrease their harmful effects and the damaging of the cell structures. Therefore, the further experimental studies can be done for this active and the other component of black pepper to treat different types of cancer diseases.

\section{Conflict of Interest}

The authors state no conflict of interest.

\section{Acknowledgments}

The calculations are carried out in the HighPerformance Computing Center and Gaussian 09W programs of Kirıkkale University. This study is supported by the Scientific Research Projects of Kirıkkale University (BAP-2016/016).

\section{References}

[1] Srinivasan, $K$., Black pepper and its pungent principle-piperine: a review of diverse physiological effects, Crit Rev Food Sci Nutr 2007;47-8 735-48.

[2] Tsai, I.L., Lee, F.P., Wu, C.C., Duh, C.Y., Ishikawa, T., Chen, J.J., Chen, Y.C., Seki, H. and Chen, I.S., New cytotoxic cyclobutanoid amides, a new furanoid lignan and anti-platelet aggregation constituents from Piper arborescens,, Planta Medica. 2005; 71 (6) 535-542.

[3] Selvendiran, K.; Padmavathi, R.; Magesh, V.; Sakthisekaran, D. Fitoterapia, Preliminary study on inhibition of genotoxicity by piperine in mice, 76, 2005;296-300.

[4] Atal, C.K.; Dubey, R.K.; Singh, Biochemical basis of enhanced drug bioavailability by piperine: evidence that piperine is a potent inhibitor of drug metabolism, J. Pharmacol Exp., 1985;232, 258262.

[5] Abdulla, M. Role of diet modification in cancer prevention, Biofactors 2000;12, 45-51.

[6] Reddy, L.; Odhav, B.; Bhoola, K.D. Natural products for cancer prevention: a global perspective, Pharmacol Ther 2003;99 1-13.

[7] Makhov, P.; Golovine, K.; Canter, D.; Kutikov,A.; Simhan, J.; Melany, M.; Corlew, Robert, G.; Uzzo and Vladimir M. , Fox Chase Cancer, Co-Administration of Piperine and Docetaxel Results in Improved Anti-Tumor Efficacy via Inhibition of CYP3A4 Activity, Kolenko Department of Urologic Oncology, Center, Philadelphia, 2012; 72(6) 661-667.

[8] Mona, A.M.; Abo-Zeid and Ayman A., F. The Anti-mutagenic Activity of Piperine against Mitomycine $\mathrm{C}$ induced Sister Chromatid Exchanges and Chromosomal Aberrations in Mice, Nature and Science, 2009;7(12). 
[9] Selvendiran, K., Sakthisekaran, D, In vivo effect of piperine on serum and tissue glycoprotein levels in benzo(a)pyrene induced lung carcinogenesis in Swiss albino mice, Pharmacology\&Therapeutics, 2006;19(2) 107-111.

[10] Yanyan, L., Max, S., Wichac, S., Schwartzb, J., Duxin, S., Implications of cancer stem cell theory for cancer chemoprevention by natural dietary compounds, Journal of Nutritional Biochemistry , 2011;22(9) 799-806.

[11] Bukhari, I. A.; Alhumayyd, M. S.; Mahesar, A. L.; Gilani, A. H. The analgesic and anticonvulsant effects of piperine in mice, Journal of Physiology and Pharmacology 2013;64(6), 789794.

[12] Samykutty, A.; Shetty, A.V.; Dakshinamoorthy, G.; Bartik, M.M.; Leon,G.J.; Webb,B.; Zheng, G.; Chen,A.; Kalyanasundaram,R. and Munirathinam, G. Piperine, a Bioactive Component of Pepper Spice Exerts Therapeutic Effects on Androgen Dependent and Androgen Independent,Prostate Cancer Cells, www.plosone.org, 2013; 8 - 6, e65889.

[13] Bharti,S., Rani,N., Krishnamurthy,B., Arya,D.S., Preclinical evidence for the pharmacological actions of naringin: a review, Planta Med. 2014;80, 437-451.

[14] Güllüoğlu,M.T., Erdoğdu,Y., Yurdakul,Ş., Molecular structure and vibrational spectra of piperidine and 4- methylpiperidine by density functional theory and ab initio Hartree-Fock calculations, Journal of Molecular tructure,2007, 834-(836),540-547.

[15] Mahalakshmi,G., Balachandran,V., Molecular structure, vibrational spectra (FTIR and FT Raman) and natural bond orbital analysis of 4Aminomethylpiperidine: DFT study, Molecular and Biomolecular Spectroscopy,2014,(131), 587598.
[16] https://www.microwaves101.com/encyclopedi as/miscellaneous-dielectric-constants.

[17] Gökalp, F., A theoritical study of Curcuma longa's anticancer agents, curcumin I and curcumin II, in blood and gas by using density functional theory (DFT) and hartree-fock (HF)Journal of Medicine and Medical Sciences, 2014; 6, 146-150.

[18] Frisch, M. J.; Trucks, G. W.; Schlegel, H. B.; Scuseria, G. E.; Robb, M. A.; Cheeseman, J. R.; Scalmani, G.; Barone, V. and et al.; Gaussian 09, Revision C. 2010;01.

[19] Zhao, P; Cao, SX; Guo, YC; Gao, P; Wang, YY; Peng, MM; Zhao, YF, Investigation of the C-N bond rotation of spirophosphorane carbamates by dynamic NMR and DFT calculation, Tetrahedron, 2015;71(46), 8738 - 8745 .

[20] Fukui, K., Science, Role of Frontier Orbitals in Chemical Reactions, 1982;218, 747-754.

[21] Pearson, R., Absolute electronegativity and hardness: applications to organic chemistry, J. Org.Chem.,54 1423-1430, (1989).

[22] Uysal,Ü.D., A DFT Study of Biogenic Amines, AKU J.Sci., 2012;12 11-24.

[23] He,D.Y., Li,Y.P., Tang,H.B., Luo,L., Ma,R.J., Wang,J.H., Phenolic compounds from the twigs and leaves of Tara (Caesalpinia spinosa), Wang,L.Q, Journal of Asian Natural Products Research, 2016;18-4, 334-338.

[24] Wanga,C., Yuana, Y., Tiana, X., Gieseking, R.L., Suna, J., Recognition of halides and Y-shaped oxoanions by carbonylchromium-based urea-like molecules: A theoretical analysis of hydrogen bonding modes, Journal of Molecular Graphics and Modelling, 2016;64 1-10. 